\title{
SelD Homolog from Drosophila Lacking Selenide-dependent Monoselenophosphate Synthetase Activity
}

\section{Britt C. Persson ${ }^{1}$, August Böck ${ }^{1 \star}$, Herbert Jäckle ${ }^{2}$ and Gerd Vorbrüggen ${ }^{2}$}

${ }^{1}$ Lehrstuhl für Mikrobiologie der Universität München Maria-Ward-Str. 1a D-80638 München, Germany

${ }^{2}$ Max-Planck-Institut für biophysikalische Chemie Abt. Molekulare Entwicklungsbiologie Postfach 2841, D-37018 Göttingen, Germany

\begin{abstract}
The isolation and molecular characterization of an invertebrate gene that encodes a homolog of the human selenophosphate synthetase 1 is described. This Drosophila gene, termed selD-like, is located in the cytogenetic interval $50 \mathrm{D} / \mathrm{E}$ on the right arm of chromosome 2. It is expressed ubiquitously throughout embryogenesis and found to be highly enriched in the developing gut and in the nervous system of the embryo.

The SelD-like from Drosophila was purified after expression in Escherichia coli. The purified protein does not catalyze the selenide-dependent ATP hydrolysis reaction and its gene does not complement a selD lesion in $E$. coli. These results and the fact that selD-like possesses an arginine residue at the position of the essential Cys17 (E. coli nomenclature) indicate that the Drosophila gene exerts a function different from that of the classical selenophosphate synthetases. Two classes of SelD proteins can therefore be differentiated. The class I proteins contain a cysteine or selenocysteine residue in the active site and display selenide-dependent selenophosphate synthetase activity. Class II proteins, including Drosophila selD-like and human selenophosphate synthetase 1 are devoid of this activity and they possess other amino acids in position 17.
\end{abstract}

(C) 1997 Academic Press Limited

\section{Introduction}

Monoselenophosphate is the key intermediate in selenium metabolism of both prokaryotes and eukaryotes. It serves as the selenium donor molecule in the conversion of seryl-tRNA ${ }^{\mathrm{Sec}}$ into selenocysteyl-tRNA ${ }^{\mathrm{Sec}}$ and in the replacement of the sulfur in 2-thiouridine in tRNA by selenium yielding 2-selenouridine (for reviews see Baron \& Böck, 1995; Stadtman, 1996). Monoselenophosphate (MSP) is the product of the reaction catalyzed by mono-selenophosphate synthetase in which the $\gamma$-phosphate group of ATP is transferred to $\mathrm{HSe}^{-}$ resulting in MSP production accompanied by the formation of inorganic phosphate and AMP (Leinfelder et al., 1990; Ehrenreich et al., 1992; Veres et al., 1992; Glass et al., 1993). A detailed kinetic analysis of the reaction mechanism of the enzyme from Escherichia coli revealed that a cysteine resi-

Abbreviations used: MSP, monoselenophosphate; IPTG, isopropyl- $\beta$,D-thiogalactopyranoside; FDH, formate dehydrogenase; PEI, polyethyleneimine. due (Cys17) is essential for catalysis (Kim et al., 1992; Veres et al., 1994).

Intriguingly, two other forms of the enzyme related to this active-site-cysteine were discovered. Analysis of the total genome sequence of Haemophilus influenzae and Methanococcus jannaschii revealed that the selD gene that codes for selenophosphate synthetase in those organisms possesses a UGA codon at the position of the E. coli Cys17, indicating that the enzyme contains a selenocysteine residue itself (Guimaraes et al., 1996; Wilting et al., 1997). Moreover, a selenocysteine-containing variant was identified in mouse and humans (Guimaraes et al., 1996). A third apparent homolog containing threonine in the respective position was identified by Low et al. (1995), also in mammalian cells (SelD1). It was shown to weakly complement a selD mutation in E. coli. When mammalian cells were transfected with this gene it stimulated Se incorporation into a selenoprotein, indicating a function in selenium metabolism.

During a sequencing project of Drosophila melanogaster another apparent selD homolog, which we designate selD-like, was discovered, representing the first sel gene found in an invertebrate. Since 
Drosophila is an excellent model organism to unravel the function of the gene products, we have initiated its genetic and biochemical analysis.

\section{Results}

\section{Isolation, structure and expression of a selD-like gene of Drosophila}

The clone carrying the apparent homolog of the gene encoding the human selenophosphate synthetase, SelD, was isolated from a Drosophila embryo cDNA library. Cytogenetic localization of the gene by in situ hybridization of the cDNA to polytene salivary gland chromosomes showed that selD-like is located in region $50 \mathrm{D} / \mathrm{E}$ on the right arm of the second chromosome (Figure 1a).

In order to determine the structure of the selDlike transcription unit, the cDNA was used as a probe to isolate genomic DNA clones from a $\lambda$ phage library. Restriction enzyme analysis of both the selD-like cDNA and its genomic counterpart, cross-hybridization experiments on Southern blots and DNA sequence comparison between cDNA and the corresponding genomic DNA allowed us to establish the structure of the gene (Figure $1 \mathrm{~b}$ and $\mathrm{d}$ ). The transcription region of selD-like expands over roughly $2 \mathrm{~kb}$. The primary selD-like transcript contains a single intron in the $5^{\prime}$ untranslated region and is spliced into a $1.5 \mathrm{~kb}$ mature transcript.

\section{Sequence analysis of the selD-like gene and comparison of the Drosophila SeID to other known SelD proteins}

Sequence analysis of the selD-like cDNA (Figure 1d) revealed that the $1540 \mathrm{bp}$ transcript contains $123 \mathrm{bp}$ of non-translated $5^{\prime}$ sequence and a single polyadenylation signal followed by a poly(A) tail extending the $193 \mathrm{bp} 3^{\prime}$ untranslated region. The putative translation start site (AGCAAGATG) corresponds to the Drosophila consensus sequence C/AAAA/CATG (Cavener, 1987). The coding sequence extends over a 1194 bp open reading frame that codes for a protein of 398 amino acid residues. This putative protein can be aligned with other SelD proteins from all evolutionary domains (Figure 2). Interestingly, the amino acid residue in the catalytic site, corresponding to cysteine 17 in the E. coli sequence (Leinfelder et al., 1990), is arginine. With one exception, namely the human SelD1, all other known SelD proteins have cysteine or a selenocysteine in this position.

\section{Expression in E. coli and purification of the Drosophila selD-like gene product (Dm-SelD)}

In the plasmid isolated from the original $\lambda \mathrm{ZAP}$ clone ( $\mathrm{pDmD}$-SelD) the selD-like gene is already under the control of the lac promoter. However, no band corresponding to the product of selD-like, Dm-SelD, could be detected in Coomassie brilliant blue-stained gels when cells harboring this plasmid were induced with $0.5 \mathrm{mM}$ IPTG. This was not unexpected, since the selD-like gene has no ribosome binding site of $E$. coli type and, hence, will probably not be translated. In $\lambda Z A P$ there is a possibility for a fusion between $l a c Z$ and the cloned gene, in $\mathrm{pDMD}-\mathrm{SelD}$, however, the reading frame of lacZ is in the -1 frame compared to that of selD. To overcome this problem and to increase the production of Dm-SelD, two plasmids were constructed. In one of them, pBP46, the expression of the selD-like gene was set under the control of both the lac promoter and the lacZ Shine-Dalgarno motif. In the other, pT7-DmD, selD-like expression was controlled by the phage T7 $\phi 10$ promoter and a synthetic Shine-Dalgarno sequence. For details on the plasmid construction, see Experimental Procedures.

The strain BL21/pT7-DmD produced large amounts of Dm-SelD at $37^{\circ} \mathrm{C}$. However, when S30 extracts were made from these cells, the Dm-SelD protein was found almost exclusively in an insoluble form. This problem was overcome by cultivation of strain BL21/pT7-DmD at $37^{\circ} \mathrm{C}$ to an $A_{600}$ of 0.5 , whereafter the culture was shifted to $+14^{\circ} \mathrm{C}$. After one hour at this temperature, production of Dm-SelD was induced by the addition of $0.1 \mathrm{mM}$ IPTG and the culture was left at $+14^{\circ} \mathrm{C}$ for approximately 30 hours. Generally about $50 \%$ of the protein produced at $+14^{\circ} \mathrm{C}$ turned out to be in a soluble form (Figure 3 ). Dm-SelD was purified from cells grown in LB medium at $+14^{\circ} \mathrm{C}$. Cells were broken and an S100 extract was made. After ammonium sulfate-precipitation the fractions containing Dm-SelD were used for further purification by ion-exchange chromatography and gel filtration (see Experimental Procedures). After the gel-filtration chromatography the Dm-SelD protein was in an apparently homogeneous state (Figure 3). $\mathrm{N}$-terminal sequencing proved its authenticity with the predicted product of selD-like and confirmed the translational start site deduced from the nucleotide sequence (see Figure 1), with the N-terminal methionine residue being cleaved off (data not shown).

\section{In vitro activity of Dm-SeID}

Selenophosphate synthetase catalyzes the reaction:

$$
\mathrm{ATP}+\mathrm{HSe}^{-} \rightarrow \text { selenophosphate }+\mathrm{AMP}+\mathrm{P}_{\mathrm{i}}
$$

The selenide-dependent hydrolysis of ATP to AMP was used as an assay for the selenophosphate synthetase activity of Dm-SelD (Ehrenreich et al., 1992). The experiments were performed under anaerobic conditions. Controls without selenide were included as well as controls with E. coli SelD instead of Dm-SelD. As shown in Figure 4, no selenophosphate was found with Dm-SelD, whereas a strong selenide-dependent activity was obtained with E. coli SelD. 


\section{In vivo activity of Dm-SelD}

One of the selenocysteine-containing proteins in E. coli is the formate dehydrogenase $\mathrm{H}(\mathrm{FDH}-\mathrm{H})$. The in vivo activity of this enzyme can be measured by providing cells with formate as the natural substrate and benzyl viologen as an artificial electron acceptor (Mandrand-Berthelot et al., 1978).

The plasmid pBP46 was used to transform the selenophosphate synthetase-deficient strain BP005

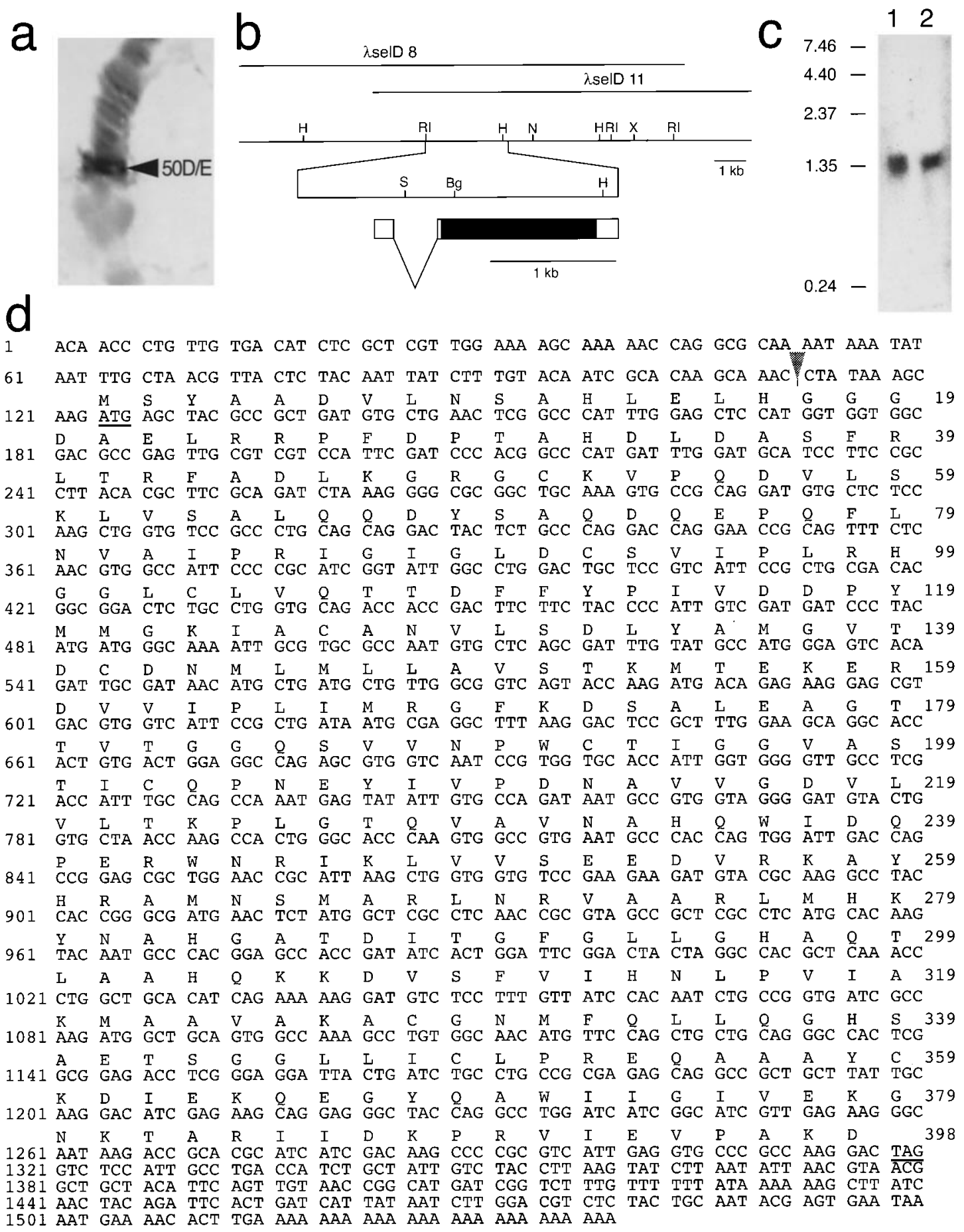

Figure 1. Molecular characterization of the Dm-selD gene locus. a, In situ hybridization of selD cDNA to polytene chromosome showing that the gene is localized within the position 50D/E at the right arm of chromosome 2. b, Position fragment, intron and exon boundaries of the transcribed region within a $16.5 \mathrm{~kb}$ fragment of genomic DNA. Bg, BglII; H, HindIII; RI, EcoRI; S, SalI; X, XbaI. c, Northern blot of total (lane 1) and poly(A) fraction (lane 2) of embryo RNA indicates a single selD-like transcript of $1.5 \mathrm{~kb}$. d, Nucleotide and protein sequence of the full-size selD-like cDNA is shown. Start and stop codons are underlined and the position of the $365 \mathrm{bp}$ intron is marked by an arrowhead; numbers of nucleotides (left) and amino acid residues (right) are shown. 
(selD::cat). To examine whether the transformants contain FDH activity they were first grown on plates in an anaerobic vial filled with $\mathrm{N}_{2}$ at $30^{\circ} \mathrm{C}$ or $37^{\circ} \mathrm{C}$, and than shifted to $+14^{\circ} \mathrm{C}$, as it was shown that the Dm-SelD is produced in a soluble form only at $14^{\circ} \mathrm{C}$. It was found that at none of these temperatures could the pBP46 or similar selD-like containing plasmids complement the FDH-H deficiency of the E. coli selD mutant. Thus, both in vitro and in vivo experiments consistently show that the SelD-like protein of Drosophila lacks selenide-dependent selenophosphate synthetase activity.

\section{Expression of selD-like in the Drosophila embryo}

The expression pattern of the selD-like gene during embryogenesis was examined by in situ hybridization with the digoxigenin-labeled selDlike cDNA probe to whole-mount preparations of embryos. Figure $5 c$ to $\mathrm{f}$ indicates that selD-like transcripts are ubiquitously distributed, at low levels, throughout the developing embryo. From early gastrulation onwards (Figure 5c), selD-like transcripts became highly enriched in the endodermal anlagen of the midgut especially in the gastric caecae and in the developing nervous system (Figure $5 \mathrm{~d}$ and e). In both locations they remain at high levels until the end of embryogenesis (Figure 5f).

\section{Discussion}

Genes coding for components required for selenocysteine biosynthesis and insertion into polypeptides have been sequenced in the last few years from a number of organisms. A comparison of the sequences revealed that SelD, the monoselenophosphate synthetase, was much more conserved during evolution than the primary structures of tRNA $^{\text {Sec }}$ (Tormay et al., 1994), SelB (Kromayer et al., 1996) or selenocysteine synthase (unpublished results). This is surprising, since SelD was thought to exclusively catalyze the transfer of a phosphate moiety from ATP to the $\mathrm{HSe}^{-}$anion, a reaction that appeared a priori not to be subject to such strong sequence constraints as those for the other sel gene products that undergo macromolecular interactions.

The SelD homologs characterized in the past few years and in this work can be divided into two major classes. Class I is characterized by the existence of a cysteine or selenocysteine residue in the active site and by catalyzing the selenide-dependent formation of MSP (Leinfelder et al., 1990; Guimaraes et al., 1996; Wilting et al., 1997). Replacement of the selenocysteine residue by cysteine decreased but did not abolish enzyme activity

SELD2 HUMAN MAEASATGACGEAMÄA. . AEESSGPAGLTLGRSFSNYRPEEROALGLSESWRLTGESGMKGUGGKVPEALLKLLAGLTRPDVRPPLGRGLVGGOEEAS SELD2 MOUSE MAEAAATGASGETMAA LVAAEGSLGPAGWSAGRSFSNYRPAEPOT LGE SPSWRLTSF SGMKGUGC KVPOET LLKLIEGLTRPALQPPLT SGLVGGOEE TV

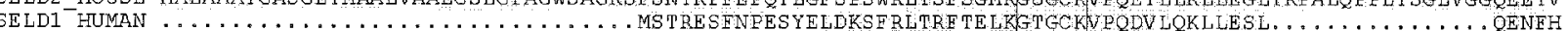
SELD1 DROME $\ldots \ldots \ldots \ldots \ldots$ MSYAADVLNSAHLELHGGGDAE LRR PFDPTȦHDLDASE RLTRFADLKGRGCKVYQDVLSKLVSAL. . . . . . . . . . OODYS

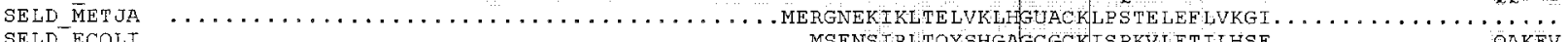
SELD ECOLI $\ldots \ldots \ldots \ldots \ldots \ldots \ldots \ldots \ldots \ldots \ldots \ldots \ldots \ldots \ldots \ldots \ldots \ldots \ldots \ldots \ldots \ldots \ldots$ IRLTOYSHGAGCGCKISPKVLETILHSE. . $\ldots \ldots \ldots$

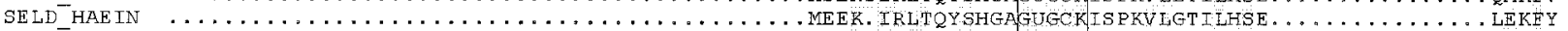

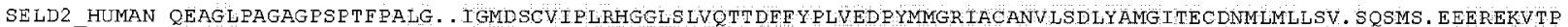

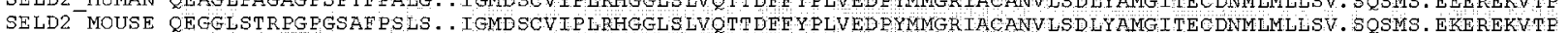

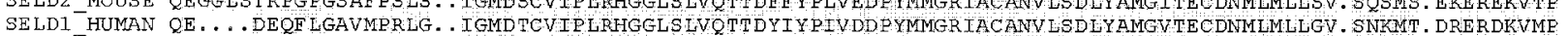

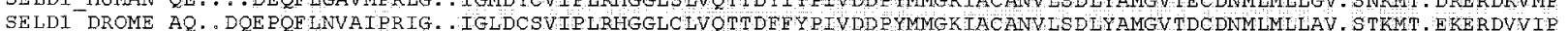

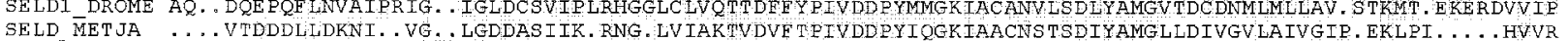

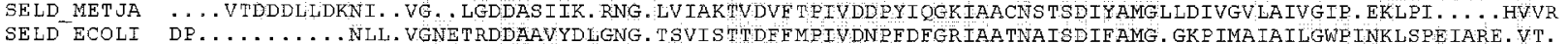

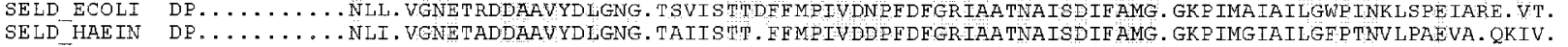

SELD2 HUMAN. LMVKGFRTAAEEGGTAVTGGQTVVNPWIIIGGVA TVVCQPNEF IMPDSAVVGDVLVLTKELGTOVAVNA. HQWLDN. PERWNKVKMVVSREE . . VELAY SELD2 MOUSE LMIKGERDAAEEGGTAVTGGQTVVNPWI I GGVATVVCQQNEETM PDSAVVGDVLVUTKE LG TQVAANA. HQWLDN. PEKWNKTKMVVSREE. . VE LAY

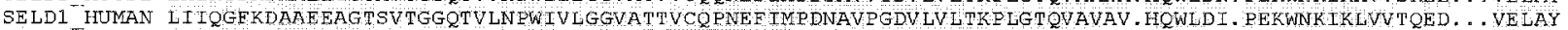

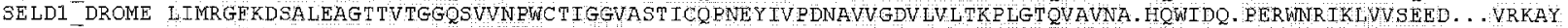

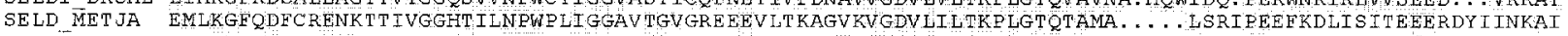

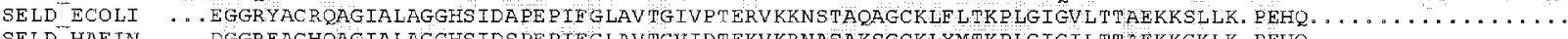
SELD_HAEIN , . DGGRFACHQAGIALAGGHSIDSPEPIFGLAVTGVTDTEKVKRNAEAKSECKLYMTKPLGIGILTTAEKKGKLK. PEHQ. . . . . . . . . . . .

SELD2_HIMAN QEAMFNMATLNRTAÄG. . LMHTFNAHAATDITGEGILGHSONLAK . . . QORNEVS . . . . . . . . FVI HNLPI IAKMAAVSKASG. RFGLL. . . .

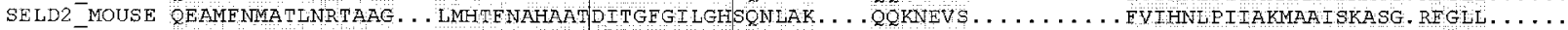

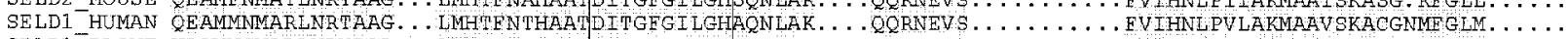

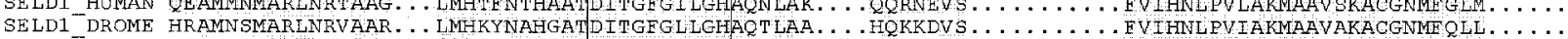

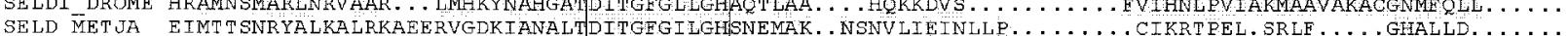
SELD ECOLI GLAT EVMCRMNIAGAS... FANTEGVKAMTDVTGEGLLGHLSEMCOGAGVOARVDYEAIPKLPGVE EYTKLGAVEGGTERNEASY.. GHLMGEMPREVRD SELD_HAEIN GLATAAMCQMNSIGSQ. . . F SQVDGVTAMT DVTGFGLUGHLIEICEGSNLSAVVESDKIKTLDGVKDY IAQGCV TGGTGRNE DSY. . GHKVGILTEEQKA

SELD2_HUMAN QGTSAETSGGLLICLPREQAARFCSEIKSSKYGEGHQAWIVGTVEKGNRTARTIDK. PRVIEVLPRGATAAVIA. PDSSNASSEDSS

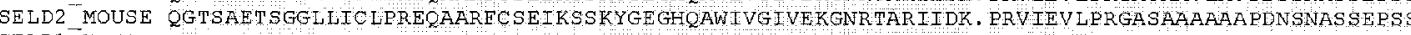

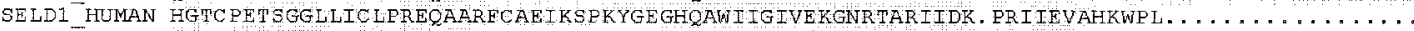
SELD1 DROME QGHSAETSGGLLICEPREQAAAYCKD . . E EKQEGYQAWI IGIVEKGNKTAR IIDK. PRVIEV PAKD $\ldots \ldots \ldots \ldots \ldots \ldots \ldots \ldots$

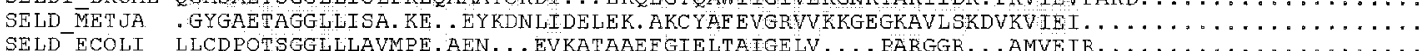
SELD HAEIN ILCDFOTSGGLIVAV. . EL. . NSVQTVIDIAKDAGIDLYEVGKLKPK. . . . SESDIVVEVK. . . . . . . . . . . . .

Figure 2. Alignment of SelD proteins from Homo sapiens (HUMAN), Drosophila melanogaster (DROME), Haemophilus influenzae (HAEIN), Escherichia coli (ECOLI) and Methanococcus jannaschii (METJA). The residue corresponding to the active-site cysteine residue (E. coli enzyme) is indicated by an arrow. The sequence motifs putatively involved in ATP binding are boxed. $\mathrm{U}$ denotes selenocysteine. 


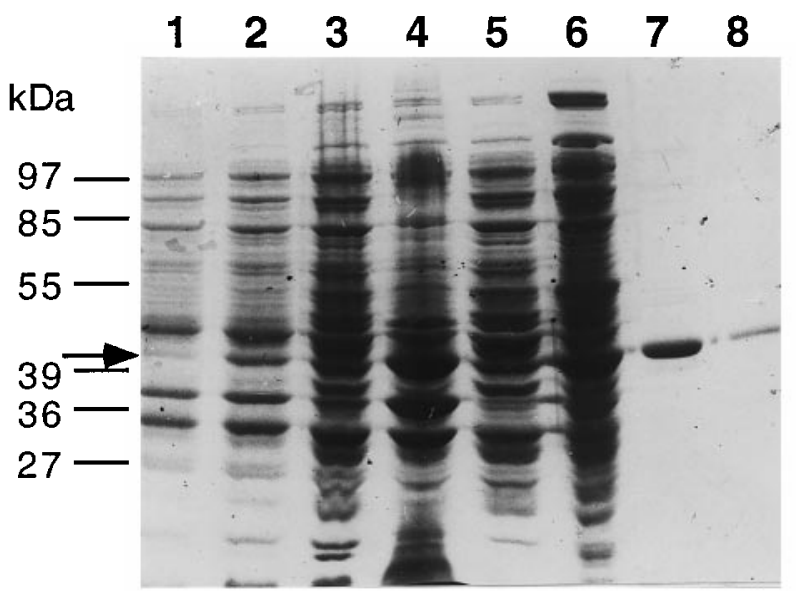

Figure 3. Purification of Dm-SelD. Lane 1, whole-cell extract before induction. Lane 2, whole-cell extract after 20 hours of induction with $0.1 \mathrm{mM}$ IPTG at $+14^{\circ} \mathrm{C}$. Lane 3, S30 supernatant. Lane 4, S30 pellet. Lane 5, S100 supernatant. Lane 6, Proteins precipitated in the 35 to $50 \%$ saturated ammonium sulfate fraction. Lane 7, DmSelD after the Q-Sepharose anion-exchange chromatography. Lane 8, Dm-SelD after the Superdex 75 gelfiltration.

(Kim et al., 1997), whereas exchange of the Cys17 residue of the E. coli enzyme against serine destroyed activity (Kim et al., 1992).

Class II SelD variants have now been demonstrated for man (Low et al., 1995) and for Drosophila in the present work. This class, although sharing a high level of sequence similarity with class I enzymes, does not have the active-site cysteine or selenocysteine residue in position 17 of the E. coli enzyme. Our results show that the SelD-like protein from Drosophila does not catalyze the selenidedependent selenophosphate formation in vitro and that its gene is unable to complete a selD deletion in an E. coli mutant in vivo. This finding correlates well with the fact that targeted replacement of

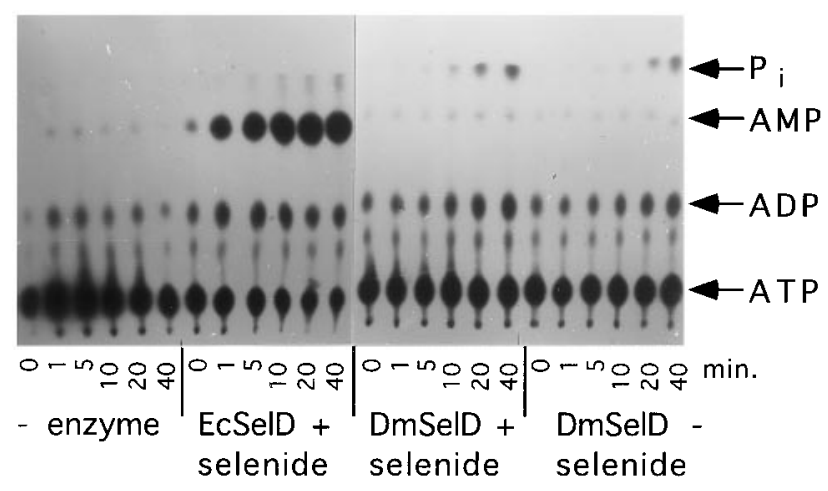

Figure 4. The selenide-dependent hydrolysis of $\left[\alpha^{32} \mathrm{P}\right]$ ATP by Dm-SelD from Drosophila and SelD from $E$. coli. Samples were taken at the time-points indicated and separated by PEI-cellulose thin-layer chromatography. The selenite added is chemically reduced by DTT in the reaction assay.

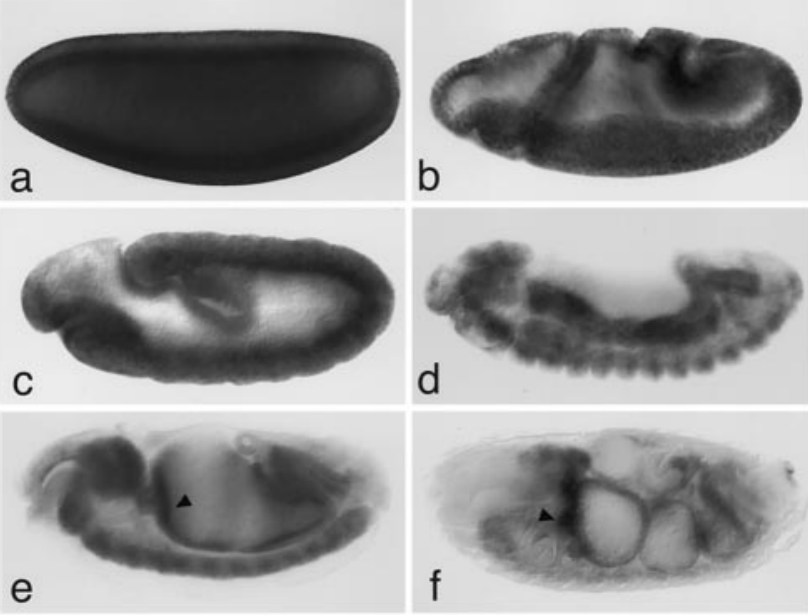

Figure 5. Expression of selD-like as revealed by in situ hybridization to whole-mount embryos preparations of embryos at various stages of Drosophila embryogenesis. a, Embryo at cellular blastoderm stage; note the strong maternal contribution. b, Embryo during gastrulation shows ubiquitous expression. c, Embryo at maximum elongated germband (stage 11) shows weak overall expression and enriched levels of transcripts in the invaginating gut anlagen and in the mesoderm. $d$, At stage 13 , transcripts are strongly expressed in the midgut and in the nervous system. e, At stage 14 transcripts continue to persist in the central nervous system and the midgut. Note the concentration of the transcript in the anlagen of the gastric caecae (arrowhead). f, In the differentiated embryo (stage 17) selD-like is expressed in the central nervous system, brain and midgut with strong enhancement in the gastric caecae (arrowhead). Stages are according to Campos-Ortega \& Hartenstein (1985).

Cys17 from E. coli SelD inactivates the enzyme completely (Kim et al., 1992) and that the human and Drosophila variants with threonine and arginine possess chemically diverse amino acids in the equivalent position. Finally, recent experiments by Kim et al. (1997) with the human class I enzyme showed that the replacement of selenocysteine by cysteine in position 17 (E. coli nomenclature) drastically reduced enzyme activity, indicating that this amino acid residue, and not some other cysteine residue is essential also for eukaryotic selenophosphate synthetase activities.

Our opinion, that class II SelD proteins are unable to catalyze the selenide-dependent selenophosphate synthesis, at first sight appears to be at variance with a report in the literature that the gene for human SelD1 complements an E. coli selD lesion (albeit weakly) and also stimulates ${ }^{75} \mathrm{Se}$ incorporation into a selenoprotein in mammalian cells (Low et al., 1995). In view of the fact that organisms that possess class II enzymes also contain a class I protein, it may well be that the SelD class II proteins have some function in selenium metabolism different from catalyzing selenidedependent selenophosphate formation. From the 
strong conservation of the ATP-binding sequence motif (Figure 2) and from the fact that mutagenesis of residues within this motif affect the stimulation of ${ }^{75} \mathrm{Se}$ incorporation into protein in transfected mammalian cells (Low et al., 1995), it appears likely that this unidentified reaction requires ATP binding and/or hydrolysis. The sequence identity of $67 \%$ between human SelD1 and Drosophila SelD supports the notion that this function is homologous in invertebrates and vertebrates. Since Drosophila genes are easily accessible to mutagenesis we shall perform targeted inactivation of selD-like to elucidate its role in vivo.

\section{Experimental Procedures}

\section{Cloning and characterization of selD-like DNA}

The selD-like cDNA clone was obtained during a low stringency screening of a $\lambda$ ZAP cDNA phage library prepared from 0 to 18 hours old embryos (Stratagene). The corresponding genomic DNA of the selD-like transcription unit (see Figure 1b) was isolated from a $\lambda$ Fix genomic Drosophila DNA library (Canton S; Stratagene). Screening of DNA libraries, the structural analysis of the selD-like gene and its transcripts as well as cytogenetic analysis by in situ hybridization to polytene chromosomes obtained from third instar salivary glands were done as described (Hartmann \& Jäckle, 1995).

Whole-mount in situ hybridizations to wild-type embryos were done with digoxigenin-labeled RNA probes as described (Tautz \& Pfeifle, 1989). All DNA fragments isolated from phages or cDNA clones were subcloned into Bluescript vectors (Stratagene). cDNA and corresponding regions in genomic DNA were sequenced by the dideoxynucleotide procedure (Sanger et al., 1977) using an automated sequencing device. The sequence has been deposited in the EMBL Data Base under the accession number A-J000672.

\section{Plasmids and strains used for purification and complementation with Dm-SelD in $E$. coli}

The bacterial strains used were the E. coli B strain BL21(DE3) hsdS, gal, which carries the gene for the phage T7 RNA polymerase under the control of the lac promoter on the $\lambda$ DE3 prophage (Studier et al., 1990), and BP005 Hfr P067, selD::cat, a derivative of KL19 (Low, 1968). For expression, two plasmids were constructed, pBP46 and pT7-DmD. The selD-like gene was put under the control of the lac promoter and lacZ ShineDalgarno sequence, by the aid of reverse PCR. The primers Dm1-rev (binding from the -1 position upstream of the ATG start codon of lacZ and reading towards the lac promoter) and Dm2-rev (binding from the ATG of selD-like and reading towards the end of the gene) were used to make a PCR product using the original pBluescript-KS + -DmD plasmid as template. The product was ligated and used to transform E. coli strain JM109. Ampicillin-resistant clones were tested for their ability to express Dm-SelD when induced with $0.1 \mathrm{mM}$ IPTG. Plasmids from positive clones were sequenced from the lac promoter to the BglII site in selD-like. The sequence downstream of the BglII site was exchanged for the same sequence from the original plasmid to make certain that the final construct pBP46 contained no sequence error caused by the PCR amplification.
In order to put the selD-like gene under the control of the phage T7 $\phi 10$ promoter, it was PCR amplified using one primer that alters the sequence at the ATG of selDlike to CATATG (NdeI) and one primer downstream of the HindIII site which is located downstream of selDlike. This PCR product was cut with NdeI and HindIII, and ligated to the pT7-7 vector (Tabor, 1994) cut with the same enzymes. As with the lac promoter construct, the region from the $\phi 10$ promoter to the BglII site in selD-like was sequenced, and the sequence from the BglII to the HindIII site was exchanged for that of the original plasmid. This places the selD-like under the control of the T7 $\phi 10$ promoter and of an artificial Shine-Dalgarno sequence (plasmid pT7-DmD).

\section{Purification of Dm-SelD}

The Dm-SelD protein was purified as follows. Cells were harvested and resuspended in buffer A $(50 \mathrm{mM}$ Tris- $\mathrm{HCl}$ ( $\mathrm{pH} \mathrm{7.5),} 50 \mathrm{mM} \mathrm{KCl}, 1 \mathrm{mM}$ DTT), and broken by three passages through a French press cell at 16,000 psi $(1 \mathrm{psi} \simeq 6.9 \mathrm{kPa})$. The lysate was centrifuged at $30,000 \mathrm{~g}$ for 30 minutes to remove cell debris, and the supernatant was further centrifuged at $100,000 \mathrm{~g}$ for two hours to obtain an S100 extract. The proteins precipitating between $35 \%$ and $50 \%$ ammonium sulfate saturation were dialyzed and used for further purification. The first chromatographic step was a Q-Sepharose anionexchange chromatography. The proteins were eluted with an $0.1 \mathrm{M}$ to $1 \mathrm{M} \mathrm{KCl}$ gradient in buffer A. Dm-SelD elutes very early in this gradient. The second chromatography step was Superdex 75 gel-filtration in buffer A containing $100 \mathrm{mM} \mathrm{KCl}$. After the gel filtration chromatography the Dm-SelD protein contained no detectable contaminating protein (Figure 3 ).

\section{Selenide-dependent ATPase assay of SeID}

As test for the selenophosphate synthetase activity of SelD the selenide- dependent hydrolysis of ATP to AMP was used (Ehrenreich et al., 1992). These experiments were performed under strictly anaerobic conditions. The reaction mixture contained $50 \mathrm{mM}$ Tris- $\mathrm{HCl}(\mathrm{pH}$ 7.5), $50 \mathrm{mM} \mathrm{KCl}, 20 \mathrm{mM}$ ATP, $5 \mathrm{mM} \mathrm{MgCl}_{2}, 2 \mathrm{mM}$ DTT, $100 \mu \mathrm{M}$ sodium selenite and $2000 \mathrm{cpm} / \mathrm{ml}\left[\alpha^{32} \mathrm{P}\right] \mathrm{ATP}$ in a total volume of $100 \mu \mathrm{l}$. Controls without selenite were included as well as controls with E. coli SelD instead of Drosophila Dm-SelD. The enzyme reaction was started by the addition of $600 \mathrm{pmol}$ of SelD. At the time-points given in Figure 4 samples $(10 \mu \mathrm{l})$ were withdrawn and the reaction stopped by addition of an equal volume of $2 \mathrm{M}$ formic acid. The samples were lyophilized and resuspended in $2 \mu \mathrm{l}$ of water; $1 \mu \mathrm{l}$ of each sample was chromatographed on polyethylenimine-cellulose thinlayer plates with non-radioactive ATP, ADP and AMP as markers. The plates were dried and autoradiographed for four hours.

\section{Acknowledgment}

We are greatly indebted to F. Lottspeich (Martinsried) for determining the N-terminal sequence. This work was supported by grants from the Deutsche Forschungsgemeinschaft (SFB 369) and the Fonds der Chemischen Industrie to A. B., by an EMBO fellowship to B. P. and by the Starke-Werner Fonds to H. J. 


\section{References}

Baron, C. \& Böck, A. (1995). The selenocysteine-inserting tRNA species: structure and function. In $t R N A$ : Structure, Biosynthesis, and Function (Söll, D. \& RajBhandary, U. L., eds), pp. 529-544, ASM Press, Washington, DC.

Campos-Ortega, J. A. \& Hartenstein, V. (1985). The Embryonic Development of Drosophila melanogaster, Springer Verlag, Heidelberg, Germany.

Cavener, D. R. (1987). Comparison of the consensus sequence flanking translational start sites in Drosophila and vertebrates. Nucl. Acids Res. 15, 13531361.

Ehrenreich, A., Forchhammer, K., Tormay, P., Veprek, B. \& Böck, A. (1992). Selenoprotein synthesis in E. coli. Purification and characterisation of the enzyme catalysing selenium activation. Eur. J. Biochem. 206, 767-773.

Glass, R. S., Singh, W. P., Yung, W., Veres, Z., Scholz, T. D. \& Stadtman, T. C. (1993). Monoselenophosphate: synthesis, characterization, and identity with the prokaryotic biological selenium donor, compound SePX. Biochemistry, 32, 12555-12559.

Guimaraes, M. J., Peterson, D., Vicari, A., Cocks, B. G., Copeland, N. G., Gilbert, D. J., Jenkins, N. A., Ferrick, D. A., Kastelein, R. A., Bazan, J. F. \& Zlotnik, A. (1996). Identification of a novel selD homolog from eukaryotes, bacteria, and archaea: is there an autoregulatory mechanism in selenocysteine metabolism? Proc. Natl Acad. Sci. USA, 93, 15086-15091.

Hartmann, C. \& Jäckle, H. (1995). Spatiotemporal relationships between a novel Drosophila stripe expressing gene and known segmentation genes by simultaneous visualization of transcript patterns. Chromosoma, 104, 84-91.

Kim, I. Y., Veres, Z. \& Stadtman, T. C. (1992). Escherichia coli mutant SELD enzymes. The cysteine 17 residue is essential for selenophosphate formation from ATP and selenide. J. Biol. Chem. 267, 19650-19654.

Kim, I. Y., Guimaraes, M. J., Zlotnik, A., Bazan, J. F. \& Stadtman, T. C. (1997). Fetal mouse selenophosphate synthetase 2 (SPS2): Characterization of the cysteine mutant form overproduced in a baculovirus-insect cell system. Proc. Natl Acad. Sci. USA, 94, 418-421.

Kromayer, M., Wilting, R., Tormay, P. \& Böck, A. (1996). Domain structure of the prokaryotic selenocysteine-specific elongation factor SelB. J. Mol. Biol. 262, 413-420.

Leinfelder, W., Forchhammer, K., Veprek, B., Zehelein, E. \& Böck, A. (1990). In vitro synthesis of selenocysteinyl-tRNA(UCA) from seryl-tRNA(UCA): involve- ment and characterization of the selD gene product. Proc. Natl Acad. Sci. USA, 87, 543-547.

Low, B. (1968). Formation of merodiploids in matings with a class of $\mathrm{rec}^{-}$recipient strains of Escherichia coli K12. Proc. Natl Acad. Sci. USA, 60, 160-167.

Low, S. C., Harvey, J. W. \& Berry, M. J. (1995). Cloning and functional characterization of human selenophosphate synthetase, an essential component of selenoprotein synthesis. J. Biol. Chem. 270, 2165921664.

Mandrand-Berthelot, M. A., Wee, M. K. K. \& Haddock, B. A. (1978). An improved method for identification and characterization of mutants of Escherichia coli deficient in formate dehydrogenase activity. FEMS Microbiol. Letters, 4, 37-40.

Sanger, F., Nicklen, S. \& Coulson, A. R. (1977). DNA sequencing with the chain terminating inhibitors. Proc. Natl Acad. Sci. USA, 74, 5463-5467.

Stadtman, T. C. (1996). Selenocysteine. Annu. Rev. Biochem. 65, 83-100.

Studier, F. W., Rosenberg, A. H., Dunn, J. J. \& Dubendorff, J. W. (1990). Use of T7 RNA polymerase to direct expression of cloned genes. Methods Enzymol. 185, 60-89.

Tabor, S. (1994). Expression using the T7 RNA polymerase/promoter system. In Short Protocols in Molecular Biology (Ausubel, F. M., et al., eds), 2nd edit, pp. 16.6-16.10, John Wiley and Sons, New York.

Tautz, D. \& Pfeifle, C. (1989). A non-radioactive in situ hybridazation method for the localization of specific RNAs in Drosophila embryos reveals translational control of the segmentation gene hunchback. Chromosoma, 98, 81-85.

Tormay, P., Wilting, R., Heider, J. \& Böck, A. (1994). Genes coding for the selenocysteine-inserting tRNA species from Desulfomicrobium baculatum and Clostridium thermoaceticum: structural and evolutionary implications. J. Bacteriol. 176, 1268-1274.

Veres, Z., Tsai, L., Scholz, T. D., Politino, M., Balaban, R. S. \& Stadtman, T. C. (1992). Synthesis of 5methylaminomethyl-2-selenouridine in tRNAs: ${ }^{31} \mathrm{P}$ NMR studies show the labile selenium donor synthesized by the selD gene product contains selenium bound to phosphorus. Proc. Natl Acad. Sci. USA, 89, 2975-2979.

Veres, Z., Kim, I. Y., Scholz, T. D. \& Stadtman, T. C. (1994). Selenophosphate synthetase. Enzyme properties and catalytic reaction. J. Biol. Chem. 269, 10597-10603.

Wilting, R., Schorling, S., Persson, B. C. \& Böck, A. (1997). Selenoprotein synthesis in Archaea: identification of an mRNA element of Methanococcus jannaschii probably directing selenocysteine insertion. J. Mol. Biol. 266, 637-641.

Edited by R. Huber

(Received 22 May 1997; received in revised form 20 August 1997; accepted 20 August 1997) 